\title{
Microfluidic Integration of Porous Photonic Crystal Nanolasers for Chemical Sensing
}

\author{
Mark L. Adams, Member, IEEE, Marko Lončar, Axel Scherer, and Yueming Qiu
}

\begin{abstract}
We have recently developed planar photonic crystal nanolasers based on porous cavity designs. High-quality factor cavities confine light within the pores of the photonic crystal and, thus, our lasers are ideally suited for the investigation of nanoscale interactions between light and matter. We have demonstrated the operation of photonic crystal lasers within different chemical solutions, embedded them into silicone microfluidic flow channels, and were able to detect refractive index changes as small as $\Delta n=0.005$. We predict that our porous nanolasers can detect refractive index changes as small as $\Delta n=8.23 \cdot 10^{-4}$.
\end{abstract}

Index Terms-Microfluidics, optical cavity, photonic crystal, polydimethylsiloxane (PDMS).

\section{INTRODUCTION}

$\mathbf{T}$ HE CONSTRUCTION of compact spectroscopic tools for the optical analysis of ultrasmall $\left(<10^{-15}\right.$ liter $)$ sample volumes remains an important goal in the development of integrated microfluidics systems. Miniaturization of appropriate light sources and detectors can enable very compact and versatile "laboratory on a chip" devices, in which many analytical functions can be monolithically combined. The porous photonic crystal cavity design permits the introduction of analyte directly into the high optical field of the laser cavity, and, due to the ultrasmall mode volume of our lasers, provides sensitivity to optical changes within femtoliter volumes.

Photonic crystals [1], [2] are microfabricated periodic nanostructures that can be designed to form stop bands for light (photonic bandgaps). Within photonic crystal mirrors, the propagation of electromagnetic waves is forbidden irrespective of the propagation direction. A structure that has recently attracted a lot of attention is a planar photonic crystal (PPC), based on a membrane with high index of refraction, suspended in air and perforated with a two-dimensional (2-D) lattice of holes [3]. One of the most promising PPC devices is a compact and efficient optical nanocavity, capable of storing electromagnetic energy in a very small volume for a long period of time [4]-[13]. Moreover, PPC cavities can be engineered to concentrate light

Manuscript received June 30, 2004; revised October 11, 2004.

M. L. Adams is with the ENSCO, Inc., Melbourne, FL 32940 USA (e-mail: adams.mark@ensco.com).

M. Lončar is with the Division of Engineering and Applied Sciences, Gordon McKay Laboratory, Harvard University, Cambridge, MA 02138 USA (e-mail: loncar@deas.harvard.edu).

A. Scherer is with the Department of Electrical Engineering, Applied Physics, and Physics, California Institute of Technology, Pasadena, CA 91125 USA (e-mail: etcher@caltech.edu).

Y. Qiu is with the In Situ Technology and Experiments Systems Section, Jet Propulsion Laboratory, California Institute of Technology, Pasadena, CA 91109 USA (e-mail: yqiu@jpl.nasa.gov).

Digital Object Identifier 10.1109/JSAC.2005.851192 in the air pores and, therefore, are natural candidates for the investigation of the interaction between light and material backfilled into the high optical field in the pores. Our porous cavity design permits the introduction of analyte directly into the high optical field of a laser cavity, and, due to the small mode volume of our lasers, permits the sensitivity to optical changes within $\approx 100$ femtoliter volumes. The introduction of matter into such cavities has a large influence on the optical signature, and the high fields obtained in the cavity can be used for spectroscopy of the cavity contents through both index of refraction changes and absorption.

\section{THEORY}

The high- $Q$ (HQ) cavity geometry that we consider here is based on a fractional edge dislocation introduced into a single defect triangular lattice photonic crystal cavity [4], [10], [14], [16]. Our PPC is constructed from an InGaAsP freestanding membrane (refractive index $n=3.4$ and thickness $d$ ) perforated with 2-D lattice of holes (periodicity $a$ and radius $r$ ). The cavity consists of a defect hole (radius $r_{\text {def }}$ ), smaller than the surrounding holes, and a row of holes that is elongated along the direction of one of the dipole modes (Fig. 1). We have analyzed our cavities using three-dimensional finite-difference time-domain (3-D FDTD) models and determined an optimal geometry $(r / a=0.3$ and $d / a=0.75)$ to efficiently confine light within the slab.

Cavities based on fractional edge dislocations support two linearly and orthogonally polarized dipole modes [4], [10] and the quality factor $(Q)$ of one of these modes can be increased by an order of magnitude over the other mode through the asymmetry of the photonic crystal along $x$ axis as seen in Fig. 1(a). The dependence of the HQ mode, which reaches lasing threshold first, and cavity geometry is described in Fig. 1(b) and (c). The dependence of the eigenfrequency and $Q$ of this mode on the distortion of the lattice $(p / a)$ and the size of the defect hole are shown. As the dislocation is extended, the spectral positions of the dipole modes are progressively detuned, and as the defect hole is enlarged, both modes shift to higher frequencies due to an increased overlap between the optical field and air.

The efficiency of an optical resonator, described by a $Q$, can be expressed as the ratio of energy stored in the cavity and the energy lost (emitted) from the cavity in one cycle. In the case of PPC cavities, there are two loss mechanisms: 1) scattering of light into the vertical direction due to coupling into the continuum of radiation modes and 2) lateral leaks through photonic crystal mirror due to the finite size of the photonic crystal. It has been shown that by adding more photonic crystal layers 
(a)

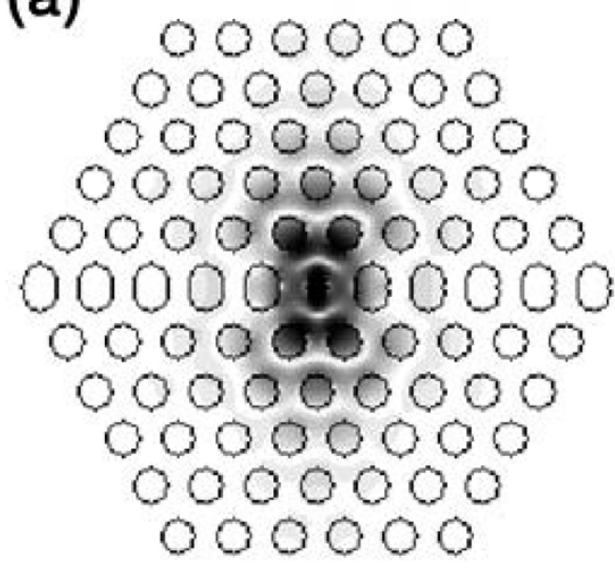

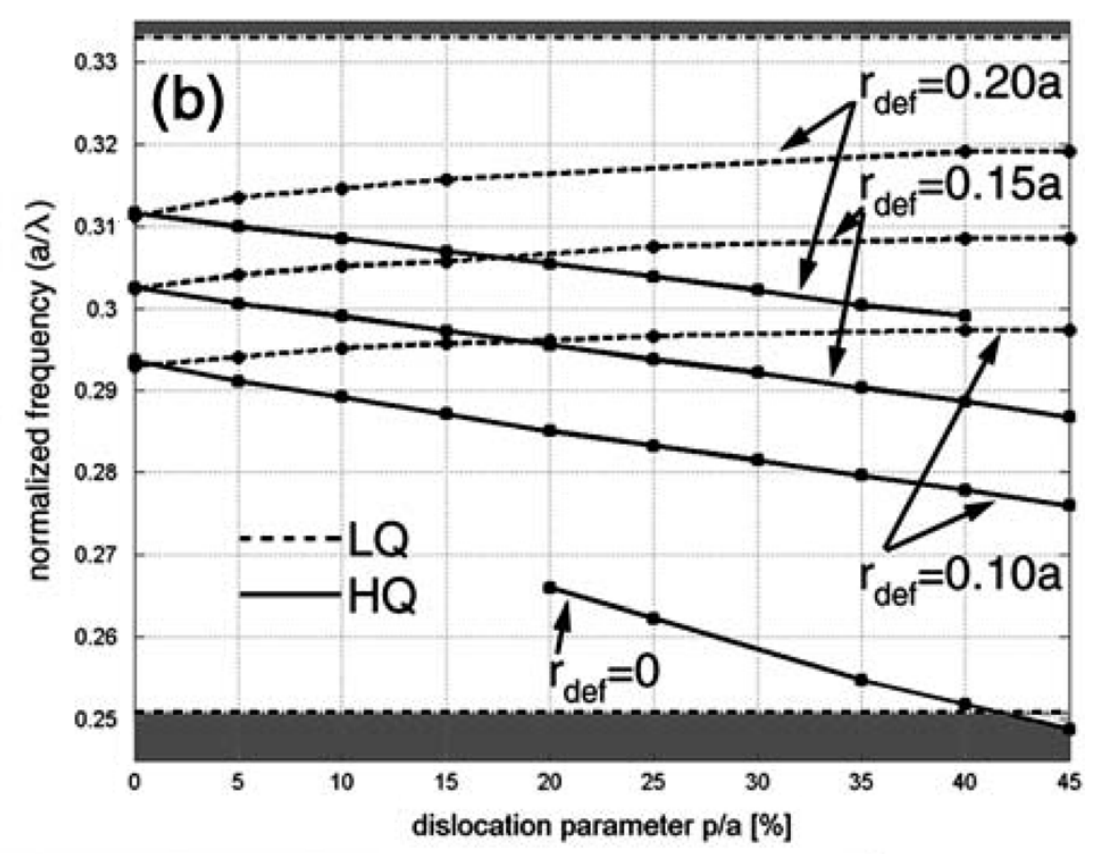

(c)

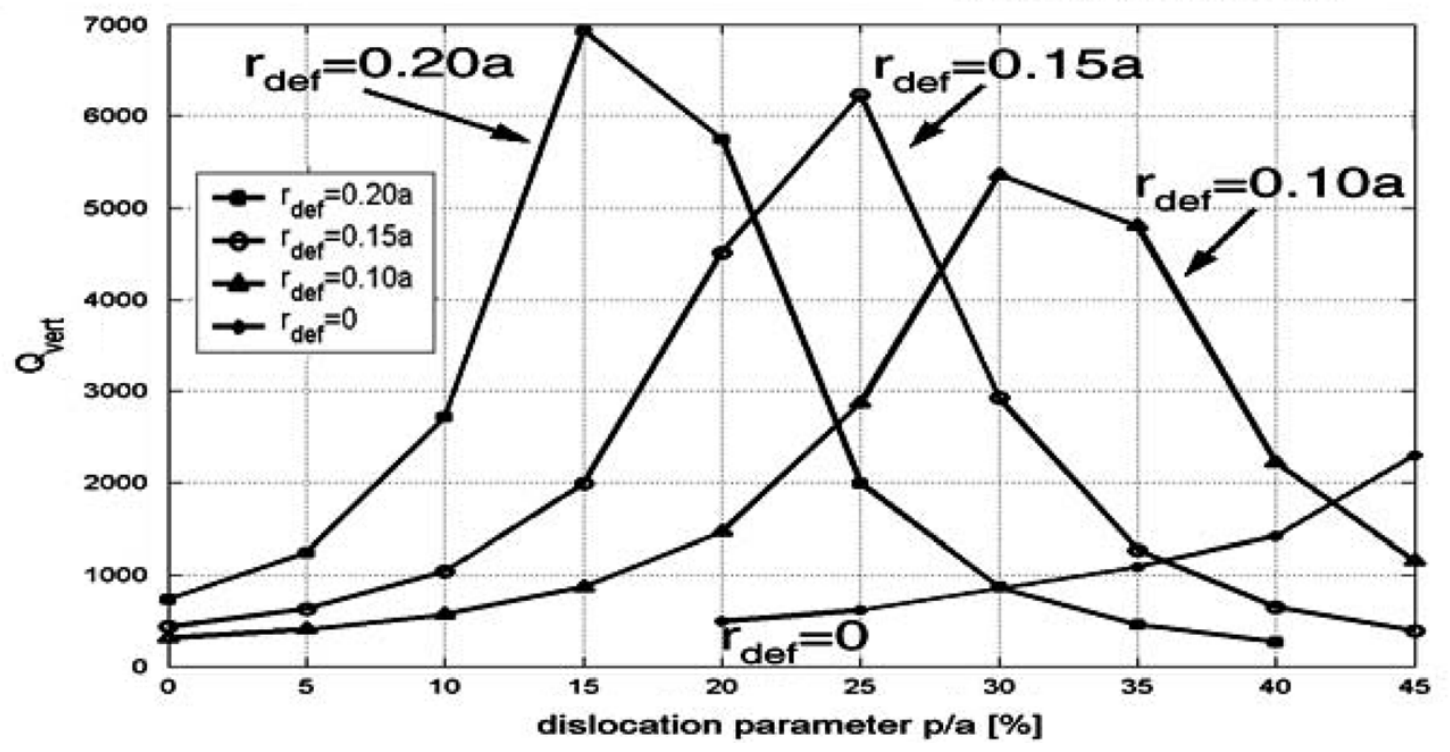

Fig. 1. (a) Field distribution ( $\left.\left|E_{\text {ampl }}\right|\right)$ of HQ dipole mode supported in the cavity. (b) Dependence of the eigenfrequencies of the two dipole modes of the cavity on the amount of dislocation introduced $(p)$, and the size of the defect hole $\left(r_{\text {def }}\right)$. The bandgap region is shown in white and it exists in the range $a / \lambda \in$ $(0.2508,0.3329)$. (c) Dependence of the vertical quality factor of the HQ mode on $p$ and $r_{\text {def }}$.

around the cavity, the latter leakage can be suppressed [15] and the $Q$ of the cavity is then dominated by vertical losses. The best $Q$ that can be achieved in the modeled cavity geometry is around 7000. However, we have chosen $r_{\text {def }}=0.15 a$ and $p=0.25 a$ for our laser sensor design $(Q \approx 6000)$, as a compromise between establishing a strong interaction between laser fields and chemicals to be analyzed (large $r_{\text {def }}$ ) and maintaining sufficient overlap between quantum well gain and cavity mode (small $r_{\text {def }}$ ).

In most common applications, PPCs membranes are suspended in air. However, it is also of interest to explore the PPC properties when the air is replaced with other materials. This is the case when PPC lasers are to be used as chemical sensors [14] or tunable filters where they are backfilled with nonlinear electrooptic polymers or liquid crystals. In Fig. 2(a), we show the dependence of the dielectric band and air-band edges on the refractive index $\left(n_{\text {env }}\right)$ of the backfilled material. As expected, the band edges are red-shifted, and the width of the bandgap decreases as $n_{\text {env }}$ increases. Structures with larger holes are more sensitive to changes in $n_{\mathrm{env}}$ and a sensitivity of over $\Delta \lambda \approx 700 \cdot \Delta n_{\mathrm{env}}$ can be expected. This suggests that a band-edge laser that operates at the air-band edge [17], [18] could detect very small changes in an ambient refractive index. However, band-edge lasers feature large mode volumes and, therefore, are not suitable for single molecule detection applications, where high spatial resolution is needed.

Therefore, it is also of interest to investigate changes in the $Q$ and eigenfrequency of the HQ mode as a laser nanocavity is backfilled with various refractive index chemicals. In Fig. 2(b). we observe that the highest $Q$ that we could hope to achieve in our cavity occurs at an ambient refractive index $n_{\text {env }}=1$ (air), and this value deteriorates as $n_{\text {env }}$ increases. This decrease 
(a)

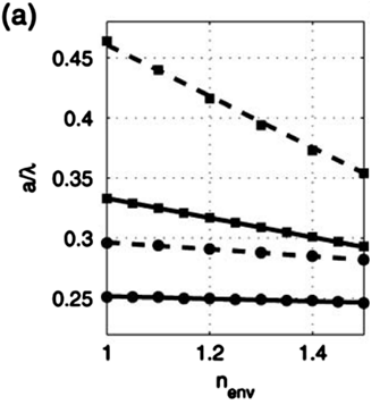

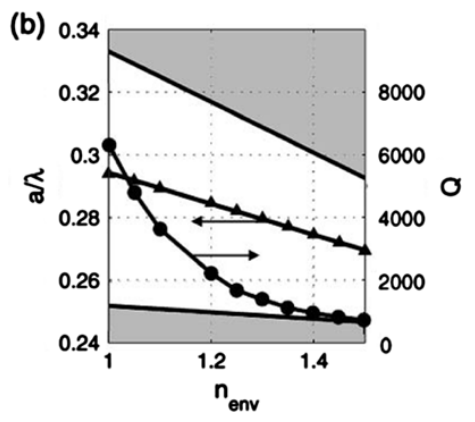

Fig 2. (a) Air-band (square) and dielectric-band (circle) edge dependence on the refractive index of environment $\left(n_{\text {env }}\right)$, in case of PPC with $r / a=0.2$ (solid line) and $r / a=0.4$ (dashed line). In both cases, bandgap closes when $n_{\mathrm{env}} \approx 2$. (b) Dependence of the eigenfrequency of cavity resonance $\left(a / \lambda=-0.0496 \cdot n_{\mathrm{env}}+0.3438\right)$ and its $Q$ factor on the refractive index of environment. Cavity geometry: $r / a=0.3, r_{\text {def }} / a=0.15$ and $p / a=25 \%$.

in $Q$ is a result of the weaker vertical confinement of light due to reduced index contrast between InGaAsP membrane and the environment, and can be compensated by increasing the thickness of the photonic crystal slab or reoptimizing the cavity design.

From linear regression of the dependence of the resonant frequency on $n_{\mathrm{env}}$, we can estimate the sensitivity of the cavity to be $\Delta \lambda \approx 266 \cdot \Delta n_{\mathrm{env}}$, where $\Delta n_{\mathrm{env}}$ is the change in refractive index and $\Delta \lambda$ is the resulting wavelength shift. If we assume that our cavity is embedded in a chemical with refractive index $n_{\mathrm{env}}=1.4$, the minimum observable wavelength shift from that cavity at $\lambda=1.55 \mu \mathrm{m}$ is $\Delta \lambda=\lambda / Q=1.55 \mathrm{~nm}(Q \approx 1000)$. This corresponds to a change in refractive index (resolution) of $\Delta n \approx 0.0056$. On the other hand, once optical gain is introduced into the cavity, as in the case of a laser spectrometer, and the linewidth of emission is further narrowed $(\Delta \lambda \approx 0.2 \mathrm{~nm})$, sensitivities of $\Delta n<0.001$ can be achieved even from cavities with modest $\mathrm{Q}$ factors.

Although the $\Delta n$ is significantly less than what can be measured by state-of-the-art measurement systems [19], the PPC offers another significant measurement tool for the lab-on-a-chip. As described above, the emission wavelength of the PPC laser is a function of the material and the geometry of the lattice. The ultimate range of emission is dictated by the material, but individual lasing lines can be geometrically tuned during fabrication. This tunability allows for a compact spectroscopic device to be made. Individual PPC lasers can then interrogate a chemical species based not only on index of refraction but also on absorption of the material. In addition, the majority of techniques used today for determining the index of refraction rely on interferometers or refractometers which cannot easily be miniaturized due to the fundamental nature of the device.

\section{FABRICATION}

Our cavities were fabricated in InGaAsP quantum-well material grown on an InP substrate using metal-organic chemical vapor deposition. Optical gain is provided by four compressively strained quantum wells, placed in the center of a 330 -nm-thick InGaAsP slab, on top of a $1-\mu$ m-thick sacrificial InP layer. The laser fabrication procedure consists of electron-beam lithography, followed by dry-and wet-etching steps
[10], [14]. 100-140-nm-thick SiON is used as an etch mask, and patterned using a $120 \mathrm{~nm}$ polymethyl methacrylate (PMMA), electron-beam (e-beam) sensitive resist layer by a reactive ionetching (RIE) procedure with $\mathrm{CHF}_{3}$ reactive gas. This SiON mask pattern is then transferred through the InGaAsP quantum wells by inductive-coupled plasma RIE etching in chlorine. The remaining SiON mask is removed by an HF dip and the InGaAsP membrane is subsequently released from the substrate by wet-etching in $4: 1 \mathrm{HCl}: \mathrm{H}_{2} \mathrm{O}$ solution at $4{ }^{\circ} \mathrm{C}$.

The final fabricated photonic crystal structures, shown in Fig. 3(a), were then embedded into polydimethylsiloxane (PDMS) microfluidic flow channels for testing. The PDMS chip was fabricated using multilayer soft lithography [20], [21]. A photoresist mold was created using SU8-2015, which had been spun at 4000 RPM to yield a height of approximately $12 \mu \mathrm{m}$. After the mold had been treated with trimethylchlorosilane (TMCS), $30 \mathrm{~g}$ of 5:1 GE RTV615 elastomer was poured onto the mold and partially cured at $80{ }^{\circ} \mathrm{C}$ for $45 \mathrm{~min}$ to create the flow channels. Once holes had been punched into the flow channels, the flow layer was ready to be aligned to the PPC layer so that one flow channel passes over several photonic crystal cavities. The pitch of the flow channels can be set to overlap the PC cavities in such a way that the alignment is much easier.

The PPC structure can not be directly embedded in PDMS since the phosphorous content of the PPC poisons the catalyst such that the elastomer never cures. In order to embed the PPC structure, it must first be coated with another material. PMMA was chosen since it does not interfere with PDMS curing and is also nondamaging for the PPC structure. The PPC structure was coated with PMMA by spinning $490 \mathrm{~K}$ molecular weight PMMA at 5000 RPM onto the structure. The PMMA was spun onto the PPC membrane side first, and then another layer of PMMA is spun onto a blank $3^{\prime \prime}$ silicon wafer. The PPC device was placed membrane first onto the wafer and the two are baked at $150{ }^{\circ} \mathrm{C}$ for $4 \mathrm{~h}$. This bonds the PPC device to the wafer. After baking the assembly was allowed to cool to room temperature and then a layer of PMMA was spun on top of the assembly to cover the backside of the PPC device. This was also baked at $150{ }^{\circ} \mathrm{C}$ for $4 \mathrm{~h}$ to drive the solvent from the PMMA. After baking the sample was allowed to cool once again and is ready for PDMS treatment.

Approximately $10 \mathrm{~g}$ of 20:1 GE RTV615 was poured onto the PPC-wafer assembly and cured at $80{ }^{\circ} \mathrm{C}$ for $8 \mathrm{~h}$. Once the PDMS was fully cured, the assembly was placed into a bath of acetone at $95{ }^{\circ} \mathrm{C}$ until the PPC structure had lifted off the silicon wafer. Since PDMS is permeable, the acetone seeps through the PDMS layer and dissolves the PMMA that bonded the PPC device to the silicon wafer. After the two had been separated, the acetone was removed and replaced with isopropyl alcohol (IPA). The sample was allowed to soak in IPA at $95^{\circ} \mathrm{C}$ for $10 \mathrm{~min}$. Afterwards, the PPC device was freed from the silicon wafer, but still encapsulated by the PDMS. The PDMS is then removed from the silicon wafer with the PPC device and placed on a hotplate at $200{ }^{\circ} \mathrm{C}$ for at least $2 \mathrm{~h}$. This was necessary to drive off any remaining solvents which may be trapped inside the PDMS. 


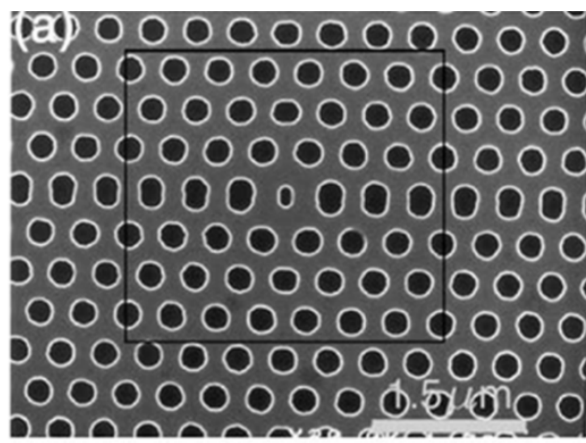

(c)

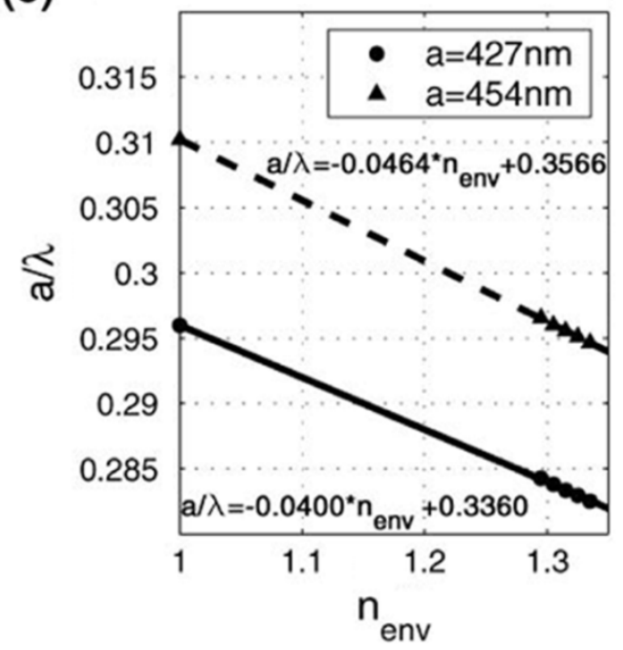

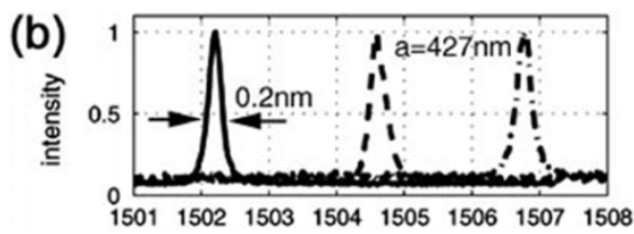

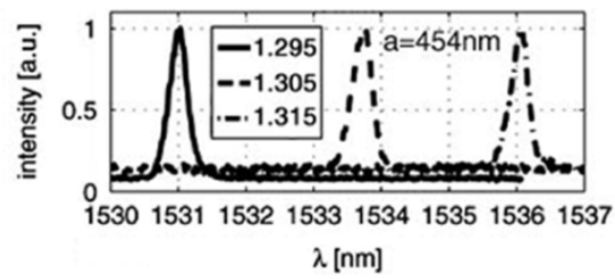

(d)

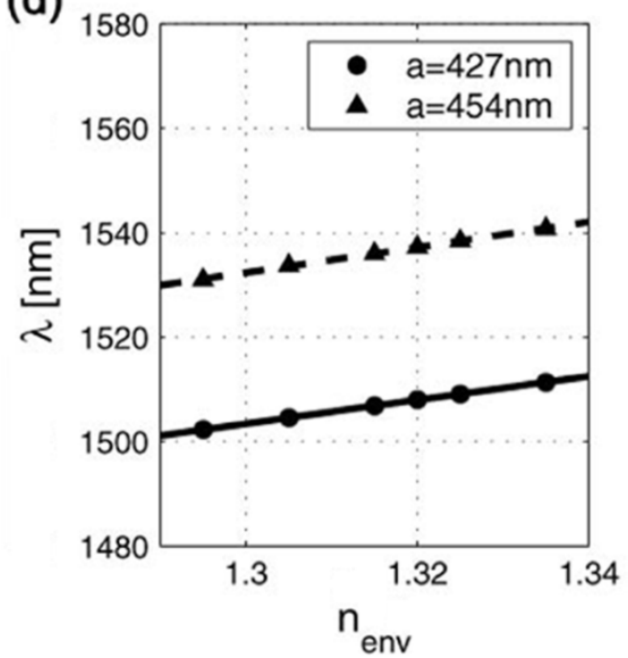

Fig. 3. (a) Fabricated structure. (b) HQ modes of two cavities experience red shifts when $n_{\text {env }}$ increases. The linewidth of the laser is $\Delta \lambda_{\mathrm{FWHM}}=0.2$ nm. Dependence of (c) $a / \lambda$ and (d) $\lambda$ of HQ mode on refractive index of the environment $\left(n_{\text {env }}\right)$, in the case of $p / a=20 \%$ cavities with two different periodicity.

In order to seal the flow layer to the PPC device layer, it was necessary to plasma treat the surface of the PPC PDMS layer. The PPC PDMS layer was placed inside a Tegal Plasmaline plasma system to perform the treatment. The layer was etched with a combination of $\mathrm{O}_{2}$ and $\mathrm{CF}_{4}$. The $\mathrm{O}_{2}$ plasma treatment is common in the literature for sealing PDMS to glass; however, the $\mathrm{CF}_{4}$ allows for a thin layer of PDMS to be removed. The recipe for etching was as follows: $20 \mathrm{sccm} \mathrm{O}, 60 \mathrm{sccm} \mathrm{O} / \mathrm{CF}_{4}$, $200 \mathrm{~W}$ forward power, $120 \mathrm{~W}$ reflected power, $30 \mathrm{~min}$. Another benefit of the plasma treatment was that it cleans the surface of the PPC membrane removing any PMMA which was not removed during the solvent treatment. After the plasma treatment, the flow layer and PPC layer can be aligned and sealed. The assembly was then cured overnight at $80^{\circ} \mathrm{C}$. A picture of the integrated PPC structure with microfluidic flow channels is shown in Fig. 4. For applications in which it is not too critical to have individual flow channels, a large reservoir, which encompasses the entire PPG structure, can be fabricated using similar methods. We have used such reservoirs defined in PDMS (depth of $100 \mu \mathrm{m})$ to perform experimental characterization of PPC nanolasers.

\section{RESULTS AND DISCUSSION}

Fabricated structures were tested at room temperature by microphotoluminescence $(\mu \mathrm{PL})[10]$, and optically pumped at $1 \%$

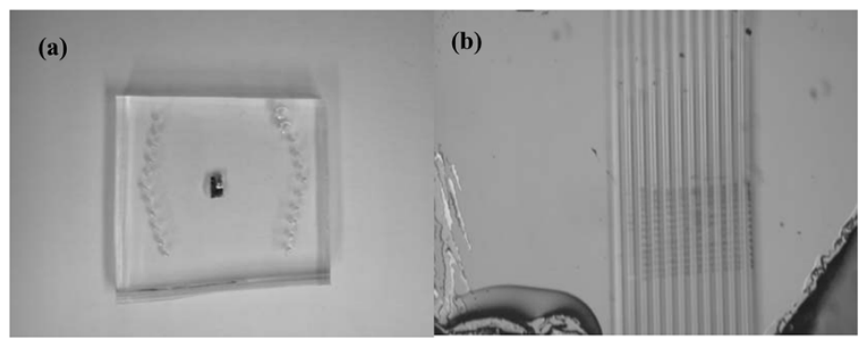

Fig. 4. (a) PPC structure embedded in a PDMS flow cell. (b) PPC lasers can be seen underneath the flow channels. The channels are $40 \mu \mathrm{m}$ wide and spaced $15 \mu \mathrm{m}$ apart.

duty cycle (30 ns pulses of $\mu$ s periodicity) with a laser diode emitting at $\lambda=830 \mathrm{~nm}$. The emission from the cavities was analyzed with an optical spectrum analyzer and imaged with an infrared (IR) camera. The simplest optical analysis can be performed when comparing wavelength shifts of the laser spectrum to measure the ambient refractive index. In this method, the sensitivity of the sensor depends on the smallest change in refractive index that can still be spectroscopically detected. In passive devices, this sensitivity is directly dependent on the width of the cavity resonance peak which in turn is determined by the cavity $Q$. However, the introduction of gain into the cavity can lead to significantly narrower linewidths than those limited by Fabry-Perot cavity $Q$ values. Thus, even smaller shifts can be 


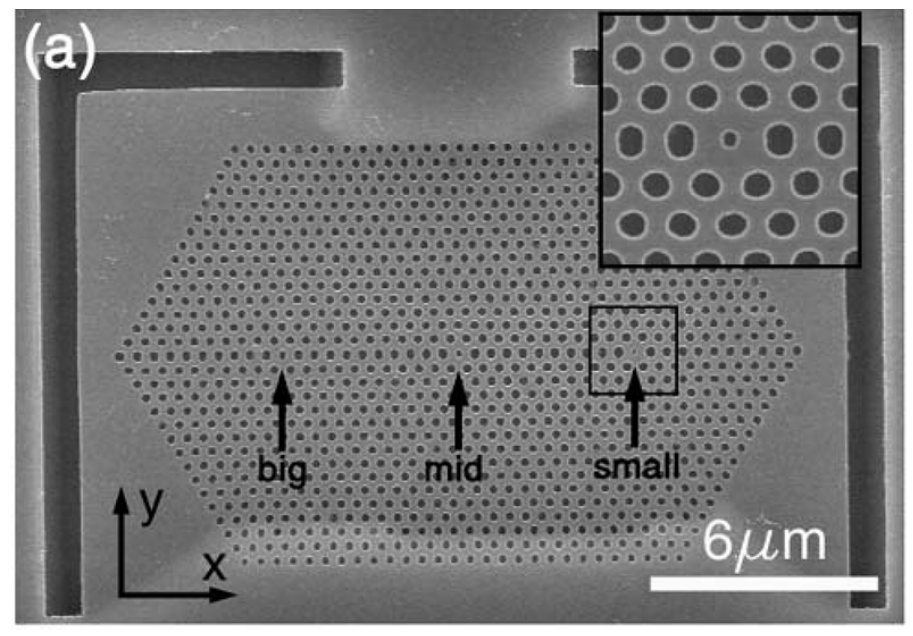

(c)

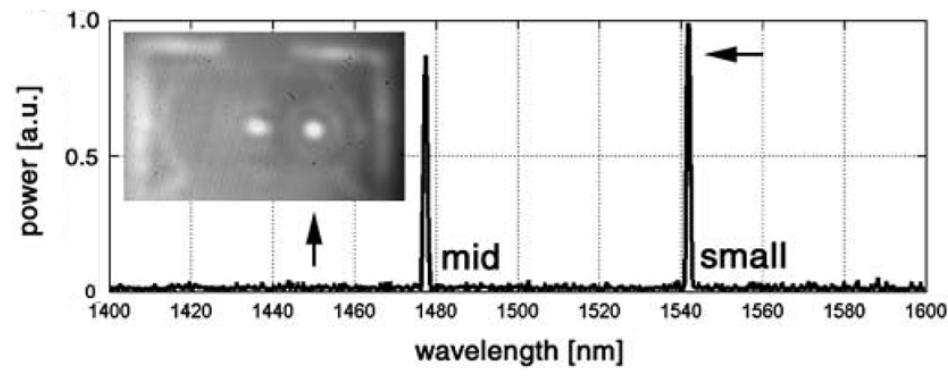

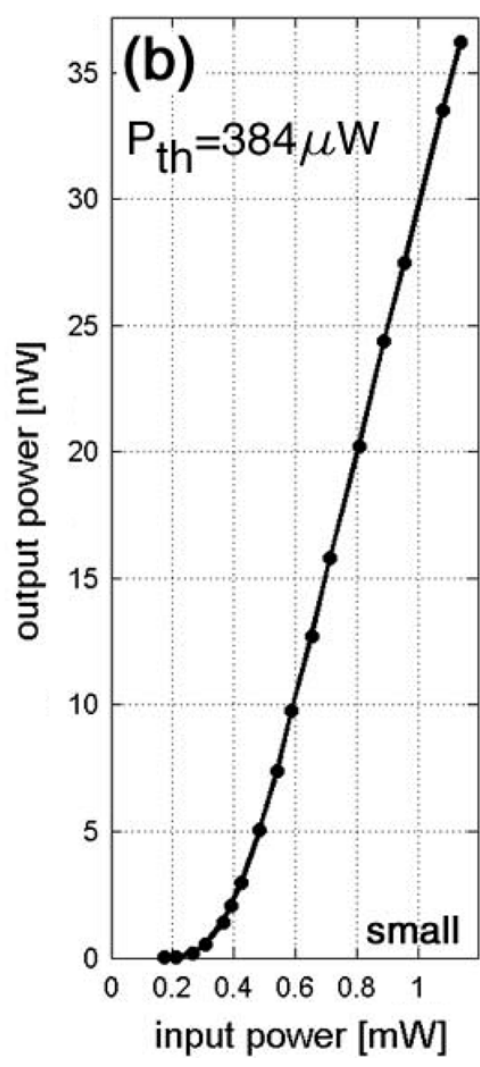

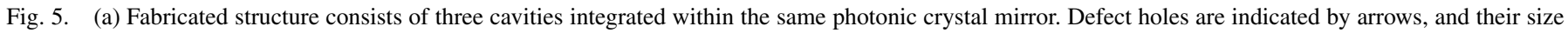

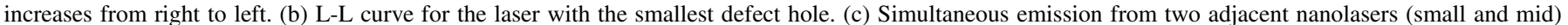

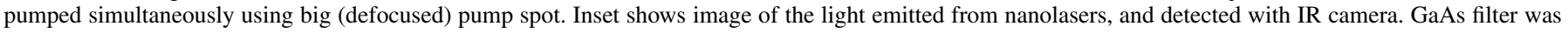
used to cut off the pump beam.

detected by monitoring lasing peaks that are spectrally narrowed through stimulated emission above laser threshold.

To test the influence of changing the ambient refractive index on such laser spectra, we have backfilled our photonic crystal lasers with refractive index calibration fluids (based on mixtures between perfluorocarbon and chlorofluorocarbon), with refractive indexes in the range between $n_{\mathrm{env}} \approx 1.295--1.335$ with step $\Delta n_{\mathrm{env}}=0.005$. The cavities were filled by pneumatically pumping the calibration fluids through the microfluidic well. After each fluid was measured the microfluidic well and the laser cavities were purged with acetone, isopropyl alcohol, and deionized water to remove any traces of the calibration fluid. The microfluidic/PPC device was then baked on a hotplate at $120{ }^{\circ} \mathrm{C}$ for $5 \mathrm{~min}$ to drive off any remaining water.

We have analyzed several different cavities and present typical results from two cavities with $p / a=20 \%$ and different lattice constants: 1) $a=427 \mathrm{~nm}$ and 2) $a=454 \mathrm{~nm}$. In Fig. 3(b), we plot the wavelength shift of HQ modes in these two cavities due to changes in $n_{\text {env }}$. In Fig. 3(c), we show the dependence of the frequency of the resonant HQ mode as a function of the refractive index of environment $\left(n_{\mathrm{env}}\right)$. It can be seen that frequency shifts depend linearly on $n_{\text {env }}$, as predicted by numerical analysis. The experimentally obtained sensitivities (slopes) are in good agreement with numerical model predictions.

While relation between $a / \lambda$ and $n_{\mathrm{env}}$ is useful for comparison between theory and experiments, relation between $\lambda$ and $n_{\mathrm{env}}$ is of more practical importance. That dependence is shown in Fig. 3(d), and we can see that the emission wavelength depends almost linearly on the refractive index of environment. The sensitivity of the laser emission wavelength on the changes in $n_{\mathrm{env}}$ is $\Delta \lambda=243 \mathrm{~nm} \cdot \Delta n_{\mathrm{env}}$ in case of structure with $a=454 \mathrm{~nm}$, and this is in good agreement with sensitivity obtained using 3-D FDTD model $\left(\Delta \lambda=266 \mathrm{~nm} \cdot \Delta n_{\mathrm{env}}\right)$. The smallest index change of index calibration fluid that we used was $\Delta n=0.005$ and we measured wavelength shifts of $\Delta \lambda=1.2 \mathrm{~nm}$ in that case. It can be seen in Fig. 3(b) that typical full-width half-maximum linewidths of lasers immersed in fluid are better than $\Delta \lambda_{\mathrm{FWHM}}=0.2 \mathrm{~nm}$, limited by the resolution of our optical spectrum analyzer. Therefore, the smallest refractive index change that we could detect by using our nanolasers is $\Delta n=8.23 \cdot 10^{-4}$ within the laser mode volume of $10^{-15}$ liter. This resolution can be further improved by introducing more gain in the cavity, pumping cavities harder, improving the fabrication of the devices and ultimately using a more sensitive readout instrument.

For many spectroscopy applications, it is desirable to construct dense arrays of ultrasmall laser cavities, tuned to different wavelengths. We have explored the integration of photonic crystal lasers into such multiwavelength sources with lithographically predetermined spectra. [Fig. 5(a)]. Optical cavities with three different defect hole sizes $\left(r_{\text {def }} / a\right)$ were incorporated within the same photonic crystal slab in order to explore the limits of such integration. The photonic crystal slab was lithographically defined ( $a=446 \mathrm{~nm}, r=128 \mathrm{~nm}$, 
$p / a=20 \%)$ and defects were introduced by modifying defect hole diameters ranging from $r_{\text {small }}=74 \mathrm{~nm}, r_{\text {mid }}=85 \mathrm{~nm}$, and $r_{\text {big }}=97 \mathrm{~nm}$. The distance between laser cavities was ten lattice periods or $\approx 4.5 \mu \mathrm{m}$.

When these cavities are individually pumped, the emission from each laser is observed only when the pump beam is focused precisely onto that nanocavity. Even slight variations in the position of the pump beam result in turning off the laser. The emission wavelength of the cavities could be tuned from $\lambda=1420 \mathrm{~nm}$ (for $r_{\text {big }}$ ) to $\lambda=1546 \mathrm{~nm}$ (for $r_{\text {small }}$ ). Fig. 5(b) shows a typical L-L curve for a laser with $r_{\text {small }}=74 \mathrm{~nm}$, and features a room temperature threshold of $P_{\mathrm{th}}=384 \mu \mathrm{W}$. It is also possible to achieve simultaneous emission from adjacent lasers when defocusing the pump beam so that the pump spot covers both cavities, as shown in Fig. 5(a) and (c). It can be seen on the infrared camera, that strong emission only originates from the positions of the two nanocavities.

As of today, the two most limiting factors for full lab-on-achip integration are the pump laser and the optical spectrum analyzer (OSA) used for readout. However, we are currently working toward a solution for both of these limitations. The most straightforward approach to eliminate the pump laser is to electrically pump the PPC devices. A significant amount of work is ongoing in this field, and we hope to demonstrate effective electrical pumping in the near future. As for the readout mechanism, a passive PPC cavity could be fabricated next to each laser cavity to couple directly to the laser cavity and interrogate its optical properties. Although, this has yet to be demonstrated, we feel that it is a viable approach for eliminating an external OSA.

\section{CONCLUSION}

In conclusion, we have characterized the operation of PPC lasers embedded in microfluidic reservoirs with different chemical solutions. These lasers were designed by 3-D modeling and fabricated in InGaAsP quantum wells by electron beam lithography followed by dry-etching. These nanocavity lasers were tested at room temperature and lasing is observed from the very localized HQ dipole mode supported by these nanocavities. Light localization is confirmed by IR camera observations of the center of the laser cavity.

In the nanolasers described here, a high-intensity optical field is localized in an air pore. Thus, this laser is fundamentally different from other semiconductor lasers, as these confine light within high-index gain material. Moreover, the ultrasmall cavity volume supports prevents mode-hopping as the air pore is backfilled with material. Therefore, photonic crystal lasers are ideally suited investigation of the interaction between light and matter at very high optical fields, and for performing spectroscopy on ultrasmall sample volumes. We show that changes in refractive index within the mode volume of the laser can be accurately measured by observing shifts in the emission wavelengths of lasers. By integrating such nanolasers with microfluidic systems defined in soft materials, it is possible to deliver of small (picoliter) quantities of reagents to sensors which can analyze even smaller (femtoliter) volumes. This new capability will enable the demonstration of a new class of highly integrated optoelectronic/microfluidic devices, with new functionality provided by placing nonlinear materials or light-emitting materials within the high field at the anti-node of an optical mode.

\section{REFERENCES}

[1] E. Yablonovitch, "Inhibited spontaneous emission in solid-state physics and electronics," Phys. Rev. Lett., vol. 58, pp. 2059-2062, 1987.

[2] S. John, "Strong localization of photons in certain disordered dielectric superlattices," Phys. Rev. Lett., vol. 58, pp. 2486-2488, 1987.

[3] T. Krauss, R. DeLaRue, and S. Brand, "Two-dimensional photonic bandgap structures operating at near infrared wavelengths," Nature, vol. 383, pp. 699-702, 1996.

[4] J. Vučković et al., "Design of photonic crystal microcavities for cavity QED,” Phys. Rev. E, vol. 6501, no. 1, p. 016 608, 2002.

[5] J. Vučković et al., "Photonic crystal microcavities for cavity quantum electrodynamics with a singe quantum dot," Appl. Phys. Lett., vol. 82, no. 15, pp. 2374-2376, 2003.

[6] K. Srinivasan and O. Painter, "Momentum space design of high-q photonic crystal optical cavities," Opt. Exp., vol. 10, p. 670, 2002.

[7] H. Ryu et al., "Square-lattice photonic bandgap single-cell laser operating in the lowest-order whispering gallery mode," Appl. Phys. Lett., vol. 80, no. 21, pp. 3883-3885, 2002.

[8] E. Miyai and K. Sakoda, "Quality factor for localized defect modes in a photonic crystal slab upon a low-index dielectric substrate," Opt. Lett., vol. 26, no. 10, pp. 740-742, 2001.

[9] H. Park et al., "Nondegenerate monopole-mode two-dimensional photonic bandgap laser," Appl. Phys. Lett., vol. 79, no. 19, pp. 3032-3034, 2001.

[10] M. Lončar et al., "Low-threshold photonic crystal laser," Appl. Phys. Lett., vol. 81, no. 15, pp. 2680-2682, 2002.

[11] T. Baba, "Photonic crystals and microdisk cavities based on GaInAsP-InP system," IEEE J. Sel. Topics Quantum Electron., vol. 3, no. 3, pp. 808-830, Jun. 1997.

[12] O. Painter, R. Lee, A. Scherer, A. Yariv, J. O'Brien, P. Dapkus, and I. Kim, "Two-dimensional photonic bandgap defect mode laser," Science, vol. 284, pp. 1819-1821, 1999.

[13] T. Yoshie, O. Shchekin, H. Chen, D. Deppe, and A. Scherer, "Quantum dot photonic crystal lasers," Elect. Lett., vol. 38, no. 17, pp. 967-968, 2002.

[14] M. Lončar, A. Scherer, and Y. Qiu, "Photonic crystal laser sources for chemical detection," Appl. Phys. Lett., vol. 82, p. 648, 2003.

[15] O. Painter, J. Vučković, and A. Scherer, "Defect modes of a two-dimensional photonic crystal in an optically thin dielectric slab," J. Opt. Soc. Amer. B, vol. 16, no. 2, pp. 275-285, 1999.

[16] T. Yoshie et al., "High quality two-dimensional photonic crystal slab cavities," Appl. Phys. Lett., vol. 79, no. 26, pp. 4289-4291, 2001.

[17] H. Ryu et al., "Very-low threshold photonic band-edge lasers from freestanding triangular photonic crystal slabs," Appl. Phys. Lett., vol. 80, no. 19, pp. 3476-3478, 2002.

[18] M. Imada et al., "Coherent two-dimensional lasing action in surfaceemitting laser with triangular-lattice photonic crystal structure," Appl. Phys. Lett., vol. 75, no. 3, pp. 316-318, 1999.

[19] S. Singh, "Refractive index measurement and its applications," Physica Scripta, vol. 65, pp. 167-180, 2002.

[20] M. A. Unger et al., "Monolithic microfabricated valves and pumps by multilayer soft lithography," Science, vol. 288, pp. 113-116, 2000.

[21] Y. N. Xia and G. M. Whitesides, "Soft lithography," Angew. Chem. Int. Ed. Engl., vol. 37, pp. 550-575, 1998.

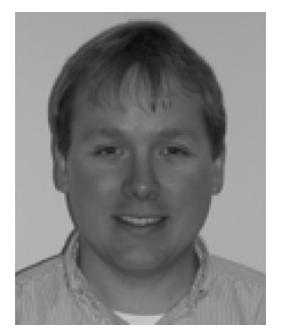

Mark L. Adams (M'03) received the B.S. degree in electrical engineering from Auburn University, Auburn, AL, in 1997, and the M.S. and Ph.D. degrees in electrical engineering with an emphasis on biophysics and nanofabrication from the California Institute of Technology (Caltech), Pasadena, in 2000 and 2004, respectively.

He has worked in industry as an Radio Frequency and Optics Engineer. He currently works as the Director of Nanobioengineering for ENSCO, Inc., Melbourne, FL (http://www.ensco.com). His interests include smart materials, organic electronics, and biologically inspired structures. 


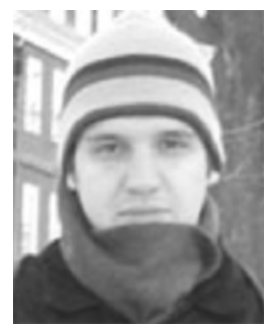

Marko Lončar received the Diploma degree in electrical engineering from the University of Belgrade, Serbia, and Montenegro, in 1997, and the M.S. and Ph.D. degrees in electrical engineering from the California Institute of Technology (Caltech), Pasadena, in 1998 and 2003, respectively.

Currently, he is a Postdoctoral Scholar at Harvard University, Cambridge, MA, working in the group of F. Capasso. His research interests are in the field of nanophotonics, including passive and active photonic crystal devices.

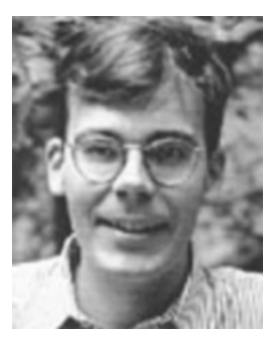

Axel Scherer received the B.S., M.S., and Ph.D. degrees from the New Mexico Institute of Mining and Technology, Socorro, in 1981, 1982, and 1985 respectively.

From 1985 to 1993 , he worked in the Quantum Device Fabrication Group, Bellcore. Currently, he is the Bernard A. Neches Professor of Electrical Engineering, Applied Physics, and Physics at the California Institute of Technology (Caltech), Pasadena. His research interests include design, fabrication and characterization of functional photonic, nanomagnetic, and microfluidic devices.
Yueming Qiu received the B.S. and M.S. degrees from the Fudan University, Shanghai, China, in 1986 and 1989, respectively, and the Ph.D. degree from the Shanghai Institute of Technical Physics, Chinese Academy of Science in 1993.

From 1994 to 1998, he was a Postdoctoral and Senior Research Associate of H. Temkin at Colorado State University and Texas Tech University. He joined the Jet Propulsion Laboratory in 1998 and currently is a Senior Member of engineering staff, responsible for MOCVD growth of III-V semiconductor materials. His research interest include the development of InP-based DFB lasers for spectroscopic applications and InAs quantum dot optoelectronic devices. 